Article

\title{
Cuscuta chinensis and C. campestris Attenuate Scopolamine-Induced Memory Deficit and Oxidative Damage in Mice
}

\author{
Ming-Kuem Lin ${ }^{1}{ }^{\circledR}$, Meng-Shiou Lee ${ }^{1}$, Hui-Chi Huang ${ }^{1}{ }^{\circledR}$, Tun-Jen Cheng ${ }^{1}$, Yih-Dih Cheng ${ }^{2}{ }^{\circledR}$ \\ and Chi-Rei $\mathrm{Wu}^{2, *(1)}$ \\ 1 Department of Chinese Pharmaceutical Sciences and Chinese Medicine Resources, China Medical University, \\ Taichung 40402, Taiwan; linmk@mail.cmu.edu.tw (M.-K.L.); leemengshiou@mail.cmu.edu.tw (M.-S.L.); \\ hchuang@mail.cmu.edu.tw (H.-C.H.); p0912789107@gmail.com (T.-J.C.) \\ 2 Department of Pharmacy, China Medical University Hospital, Taichung 40402, Taiwan; \\ M99553@mail.cmuh.org.tw \\ * Correspondence: crw@mail.cmu.edu.tw; Tel.: +886-4-2205-3366
}

Academic Editors: Paula B. Andrade and Patrícia Valentão

Received: 9 October 2018; Accepted: 21 November 2018; Published: 22 November 2018

\begin{abstract}
The seeds of Cuscuta chinensis Lam. and C. campestris Yuncker have been commonly used as Chinese medical material for preventing aging. Our previous studies have found that $C$. chinensis and $C$. campestris possess anti-inflammatory activities in rodents. However, their other biological activities, such as memory-improving properties, have not yet been explored. In the present study, we examined the memory-improving effects of the extracts of $C$. chinensis and $C$. campestris on scopolamine (SCOP)-induced memory deficit and explored their underlying mechanism in mice. Both Cuscuta species improved SCOP-induced memory deficits in the passive avoidance test, elevated plus-maze, and spatial performance test of the Morris water maze in mice. In addition, compared with mice injected with SCOP, mice pretreated with both Cuscuta species stayed for a longer time on the platform for the probe test of the Morris water maze. Moreover, both Cuscuta species reduced brain acetylcholinesterase activity and malondialdehyde levels that were increased by SCOP, and the species restored the activities of antioxidant enzymes (superoxide dismutase and catalase) and the levels of glutathione that were decreased by SCOP in the brains of mice. Both Cuscuta species further decreased brain interleukin-1 $\beta$ and tumor necrosis factor- $\alpha$ levels that were elevated by SCOP. We demonstrated that both Cuscuta species exhibited a protective activity against SCOP-induced memory deficit, cholinergic dysfunction, oxidative damage, and neuroinflammation in mice, and C. campestris has better potential than $C$. chinensis. In addition, we provided evidence that the seeds of $C$. campestris can be used as Cuscutae Semen in Traditional Chinese Medicine.
\end{abstract}

Keywords: Cuscutae Semen; Cuscuta campestris; Cuscuta chinensis; memory-improving properties; scopolamine

\section{Introduction}

Alzheimer's disease (AD), a progressive neurodegenerative disorder, endures major clinical symptoms such as behavioral and cognitive decline. These symptoms are caused by three major and neuropathological mechanism including the degeneration of cholinergic neuronal circuits in the basal forebrain $(\mathrm{BF})$, extracellular amyloid $\beta$ peptide $(\mathrm{A} \beta)$ deposition, and intracellular neurofibrillary tangles formation from hyperphosphorylated tau protein [1]. Recent studies have speculated that oxidative stress and/or inflammatory processes are also important biochemical factors in the above neuropathological characterization [2]. Accumulation of free radical damage and alterations in the 
activities of brain antioxidant enzymes such as superoxide dismutase (SOD) and catalase have been observed in AD patients [3]. When oxidative damage to lipids and proteins increased, the severity of cholinergic neuronal loss and deficits in memory and behavior also increased [4,5]. Learning and memory processes usually involve the activation of neurotransmitters such as acetylcholine and monoamines [6]. According to Myhrer's meta-analysis of studies on four behavioral tasks [7], the acetylcholinergic system has a high influence on learning and memory. Scopolamine (SCOP), a muscarinic receptor antagonist, impairs learning and memory in rodents and humans mainly by decreasing cholinergic activity $[8,9]$. Some recent reports have indicated that SCOP causes cerebral oxidative damage and neuroinflammation, which are the same as the neuropathological mechanism of AD $[10,11]$. Hence, SCOP has been used to induce amnesia and/or dementia in experimental rodent models.

Cuscuta chinensis Lam. and C. campestris Yuncker belong to the genus Cuscuta of the Cuscutaceae family. The seeds of $C$. chinensis (Cuscutae Semen), which is recorded in the famous book Shen Nong's Herbal and other Chinese traditional pharmacopoeias, have been commonly used as medicine to nourish the kidney and liver in Traditional Chinese Medicine. However, C. campestris Yuncker, which is widely distributed in the wild, is not recorded in pharmacopoeias in Taiwan. Many studies have shown that Cuscuta species have various biological activities, including antibacterial, antimicrobial, antioxidant, anti-inflammatory, antiproliferative, hepatoprotective, and antiulcer activities [12-14]. C. chinensis reduced the levels of nitric oxide and malondialdehyde (MDA) by increasing the activities of antioxidant enzymes in the liver [13], and it reduced the levels of inflammatory mediators and proinflammatory cytokines in lipopolysaccharide-stimulated BV-2 microglia and RAW264.7 cells [15,16]. C. chinensis also improved the learning-memory ability in AD mice with hypoendocrinism [17]. Flavonoids of $C$. chinensis exerted the protective effects on cerebral ischemia-reperfusion injury by inhibiting inflammation in brain tissue [18]. C. campestris exhibited antioxidant activity in vitro as well as antifungal activity [19], and it reduced the MDA level by increasing the activities of antioxidant enzymes in the liver [20]. These findings reveal that both $C$. chinensis and C. campestris possess antioxidant and anti-inflammatory activities. Previous studies have shown that quercetin 3-O- $\beta$-D-apiofuranosyl-(1-2)- $\beta$-D-galactoside, hyperoside, quercetin, and kaempferol are the major compounds in the seeds of $C$. chinensis, and the same compounds, excluding kaempferol, are present in the seeds of $C$. campestris. However, the contents of these compounds are different in the seeds of the two species $[13,16,21]$. This study evaluated whether $C$. campestris can be a substitute for C. chinensis for preventing aging. Due to the pathological mechanisms of AD, including oxidative stress and neuroinflammation, $C$. chinensis and C. campestris might possess brain neuroprotective effects. Therefore, this study examined and compared their efficacies and explored their mechanisms for their antioxidant and anti-inflammatory potential in SCOP-induced memory deficits in mice.

\section{Results}

\subsection{Effects of C. chinensis and C. campestris Extracts on Locomotor and Exploratory Activities in} SCOP-Treated Mice

Compared with the control group, SCOP increased the movement time and distance and then accelerated the movement velocity (Figure 1 ). The C. chinensis or C. campestris extract at any dose did not alter the higher locomotor activity caused by SCOP (Figure 1). SCOP slightly increased the number of entries and the time spent in the hole, but no significant difference was observed between the SCOP and control groups. However, SCOP decreased the ratio of the time spent to the entry number in the hole test in mice (Figure 2). The $C$. chinensis or $C$. campestris extract did not change the behavioral alteration caused by SCOP (Figure 2). 
(A) Movement time

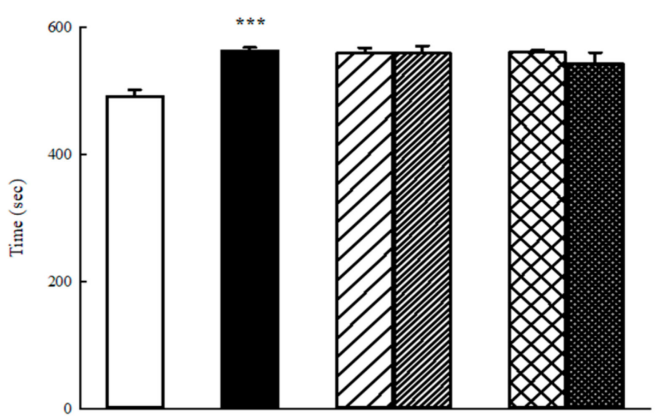

(B) Movement distance

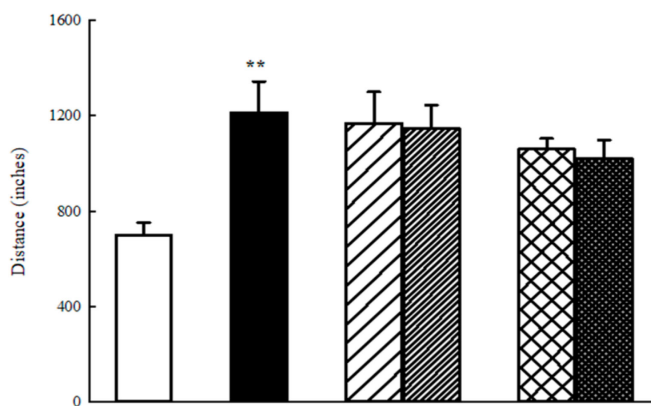

(C) Velocity

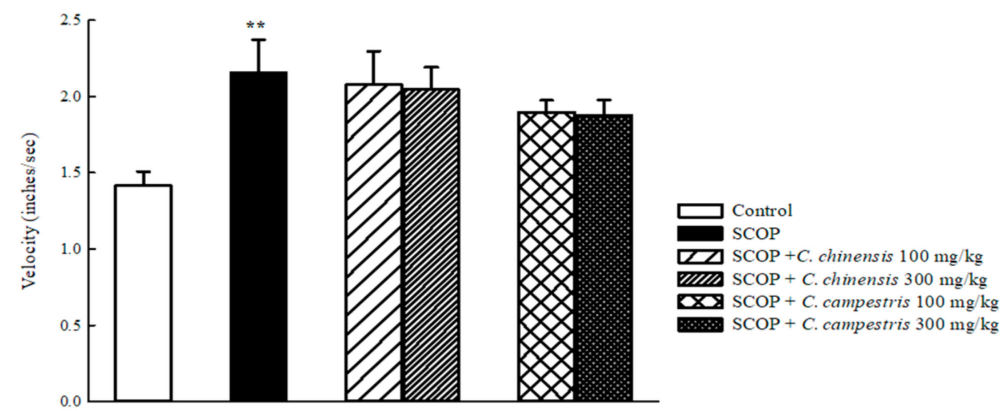

Figure 1. The effects of Cuscuta chinensis and C. campestris extracts (100 and $300 \mathrm{mg} / \mathrm{kg}$, po) on (A) movement time, (B) movement distance, and (C) velocity in scopolamine (SCOP, $1 \mathrm{mg} / \mathrm{kg}$, ip)-treated mice. Columns indicate mean $\pm \operatorname{SEM}(n=7) .{ }^{* *} p<0.01,{ }^{* * *} p<0.001$ compared with the control group.

(A) Entry into hole

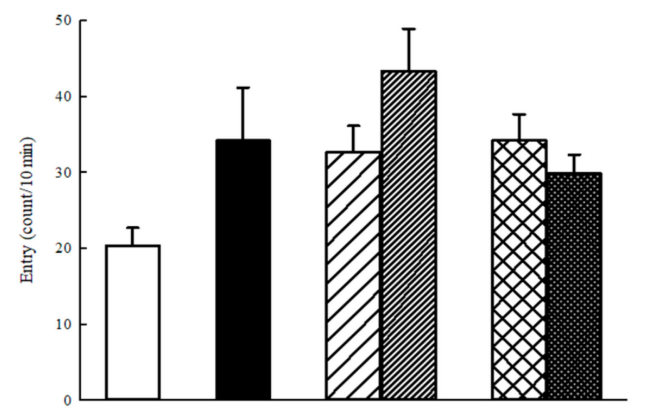

(C) Ratio
(B) Time spent in hole

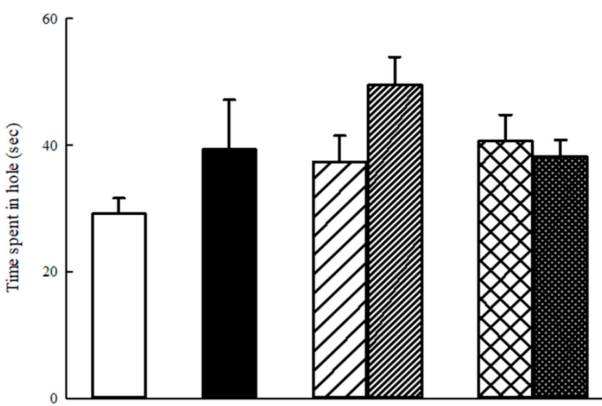

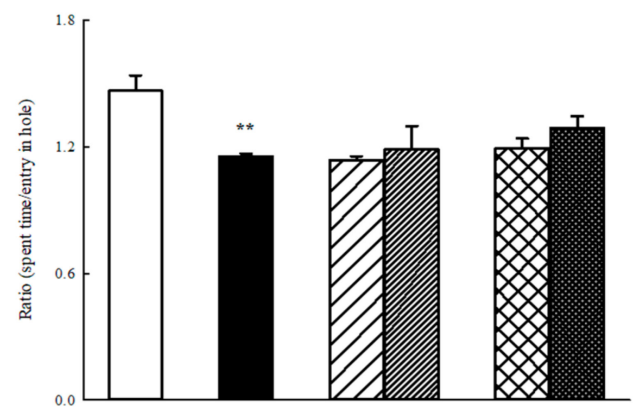

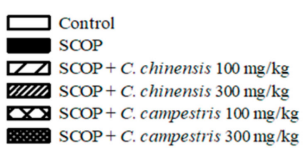

Figure 2. The effects of Cuscuta chinensis and C. campestris extracts (100 and $300 \mathrm{mg} / \mathrm{kg}$, po) on (A) entries into hole, (B) time spent in hole, and (C) ratio of the time spent to the entry number in scopolamine (SCOP, $1 \mathrm{mg} / \mathrm{kg}$, ip)-treated mice. Columns indicate mean $\pm \operatorname{SEM}(n=7) .{ }^{* *} p<0.01$ compared with the control group. 


\subsection{Effects of C. chinensis and C. campestris Extracts on Memory Deficits in SCOP-Treated Mice}

Compared with the control group, SCOP shortened the step-through latency (STL) of the retention trial (Day 2 of the passive avoidance test) (Figure 3A). The C. chinensis or C. campestris extract at $300 \mathrm{mg} / \mathrm{kg}$ prolonged the STL of the retention trial that was shortened by SCOP (Figure 3A). However, no difference was observed in the STL of the acquisition trial (Day 1 of the passive avoidance test) among the control, SCOP-treated, and C. chinensis or C. campestris extract-treated groups (Figure 3A). SCOP increased the ratio of the transfer latency (TL) of the retention session to the TL of the acquisition session in the elevated plus-maze (Figure 3B). The C. chinensis or C. campestris extract at 100 and $300 \mathrm{mg} / \mathrm{kg}$ decreased the ratio of the TL of the retention session to the TL of the acquisition session that was increased by SCOP (Figure 3B).
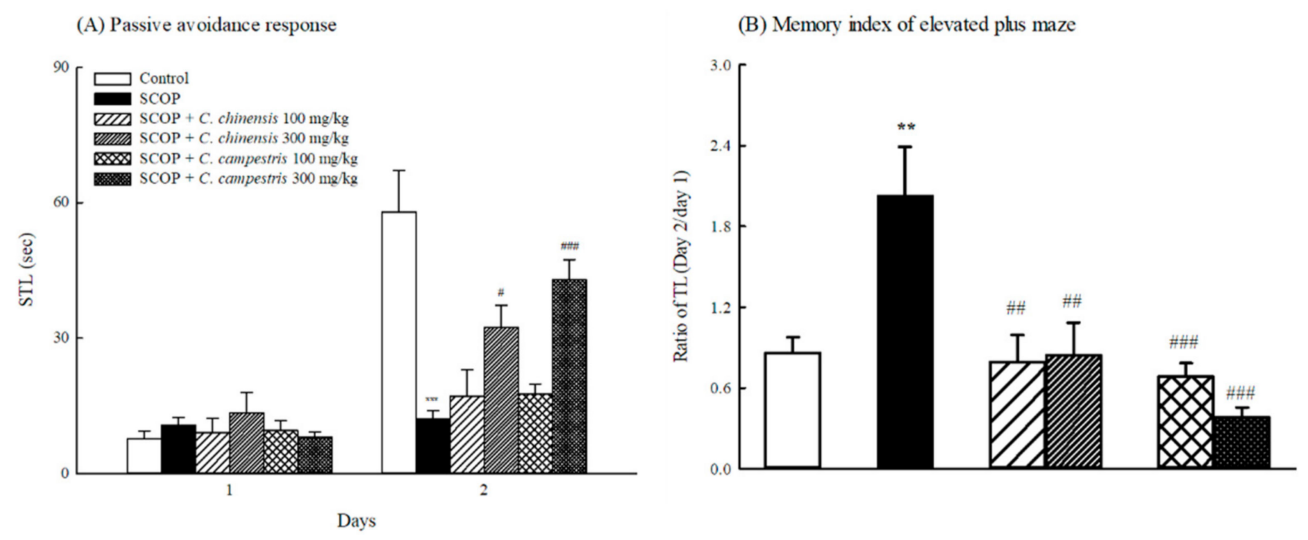

Figure 3. The effects of Cuscuta chinensis and C. campestris extracts (100 and $300 \mathrm{mg} / \mathrm{kg}$, po) on (A) passive avoidance response and (B) memory index on elevated plus-maze in scopolamine (SCOP, $1 \mathrm{mg} / \mathrm{kg}$, ip)-treated mice. Columns indicate mean $\pm \operatorname{SEM}(n=7) .{ }^{* *} p<0.01,{ }^{* * *} p<0.001$ compared with the control group. \# $p<0.05$, \#\# $p<0.01$, \#\#\# $p<0.001$ compared with the SCOP group.

Mice injected with SCOP took a longer escape latency to reach the platform in the spatial performance test (Days 2-4 of the Morris water maze (MWM)) than those in the control group (Figure 4A). The C. chinensis extract at 100 and $300 \mathrm{mg} / \mathrm{kg}$ only shortened the longer escape latency, which was caused by SCOP, taken to reach the platform on Day 4 of the spatial performance test in MWM. The C. campestris extract at 100 and $300 \mathrm{mg} / \mathrm{kg}$ shortened the longer escape latency, which was caused by SCOP, taken to reach the platform on Days 3 and 4 of the spatial performance test in MWM (Figure 4A). Moreover, compared with the control group, SCOP decreased the time spent on the platform in the probe test of MWM (Figure 4B). The C. chinensis or C. campestris extract only at $300 \mathrm{mg} / \mathrm{kg}$ increased the time spent on the platform in the probe test of MWM (Figure 4B). Furthermore, SCOP accelerated the swimming velocity compared with that in the control group (Figure 4C). Compared with the SCOP group, SCOP-injected groups treated with the $C$. chinensis or C. campestris extract did not alter the swimming velocity in MWM (Figure 4C). 
(A) Time spent to find the hidden platform

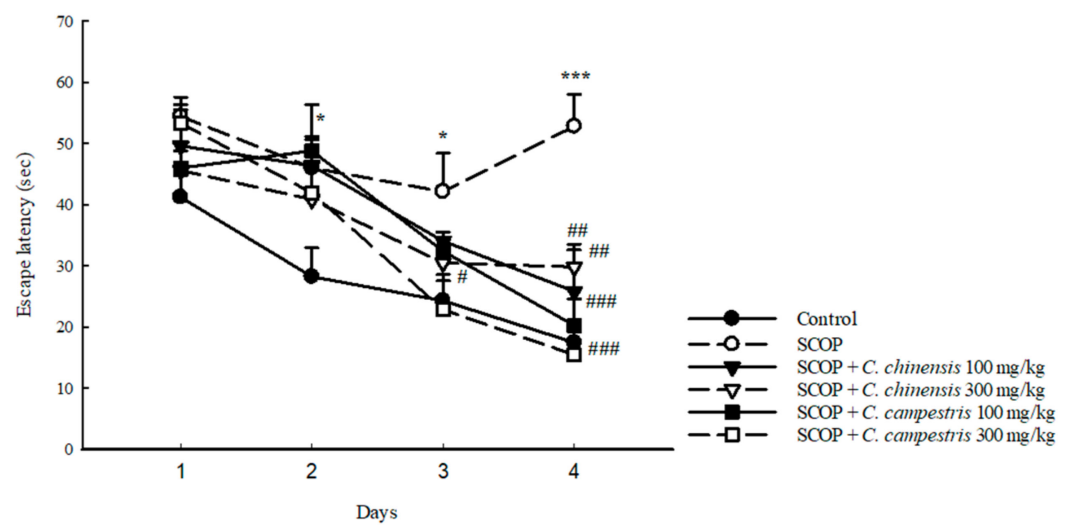

(B) Probe test

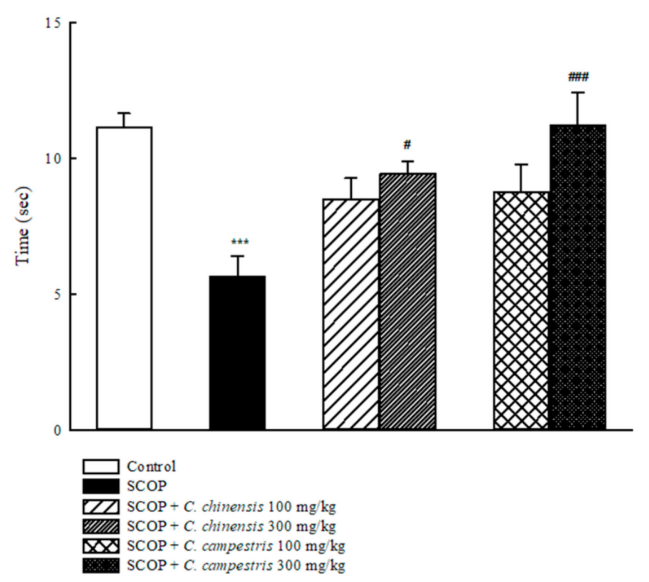

(C) Velocity

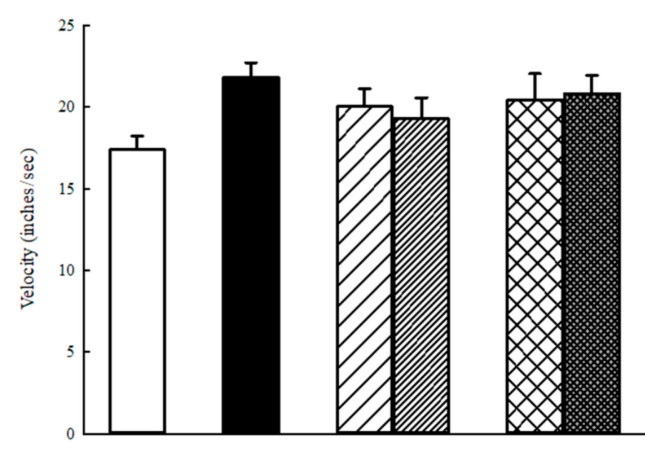

Figure 4. The effects of Cuscuta chinensis and C. campestris extracts (100 and $300 \mathrm{mg} / \mathrm{kg}$, po) on (A) swimming time on Days 1-4 of MWM, (B) probe test of MWM, and (C) swimming velocity in scopolamine (SCOP, $1 \mathrm{mg} / \mathrm{kg}$, ip)-injected mice. Columns indicate mean $\pm \operatorname{SEM}(n=7) .{ }^{*} p<0.05$, *** $p<0.001$ compared with the control group. \# $p<0.05$, \#\# $p<0.01$, \#\#\# $p<0.001$ compared with the SCOP group.

2.3. Effects of C. chinensis and C. campestris Extracts on Brain Acetylcholinesterase Activities in SCOP-Treated Mice

SCOP increased brain acetylcholinesterase (AChE) activities in mice (Figure 5). The C. chinensis extract at any dose did not alter brain $\mathrm{AChE}$ activities that were increased by SCOP. The $C$. campestris extract at $300 \mathrm{mg} / \mathrm{kg}$ decreased brain AChE activities that were increased by SCOP (Figure 5). 


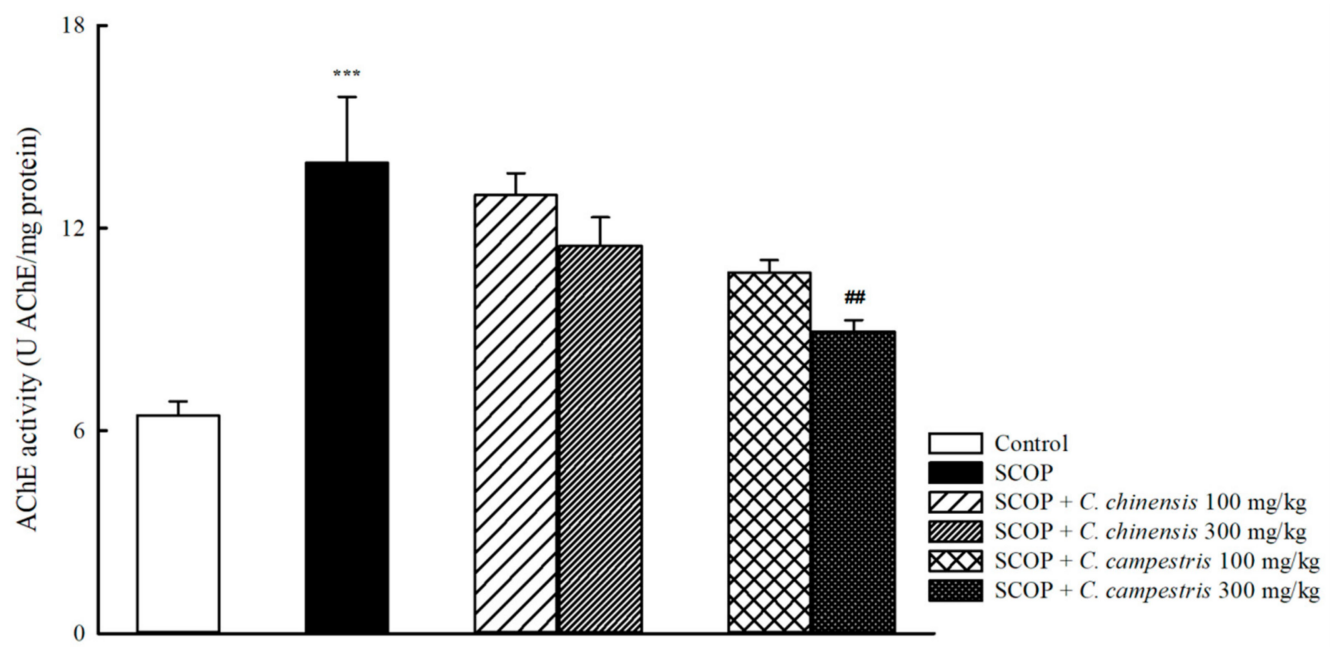

Figure 5. The effects of Cuscuta chinensis and C. campestris extracts (100 and $300 \mathrm{mg} / \mathrm{kg}$, po) on brain acetylcholinesterase (AChE) activities in scopolamine (SCOP, $1 \mathrm{mg} / \mathrm{kg}$, ip)-treated mice. All the brain tissues were collected from the tested mice and then homogenized to obtain the total brain homogenates. AChE activities in the total brain homogenates were subsequently examined. Columns indicate mean \pm $\operatorname{SEM}(n=7) .{ }^{* * *} p<0.001$ compared with the SCOP group. \#\# $p<0.01$ compared with the SCOP group.

\subsection{Effects of C. chinensis and C. campestris Extracts on Brain Oxidative Damage in SCOP-Treated Mice}

SCOP decreased SOD and catalase activities and glutathione (GSH) levels and then increased MDA levels in the brains of mice (Figure 6). The C. chinensis or C. campestris extract at $300 \mathrm{mg} / \mathrm{kg}$ increased brain SOD and catalase activities that were decreased by SCOP. The $C$. chinensis or C. campestris extract at $300 \mathrm{mg} / \mathrm{kg}$ decreased MDA levels that were elevated by SCOP (Figure 6). However, only the C. campestris extract at $300 \mathrm{mg} / \mathrm{kg}$ increased brain GSH levels that were decreased by SCOP.
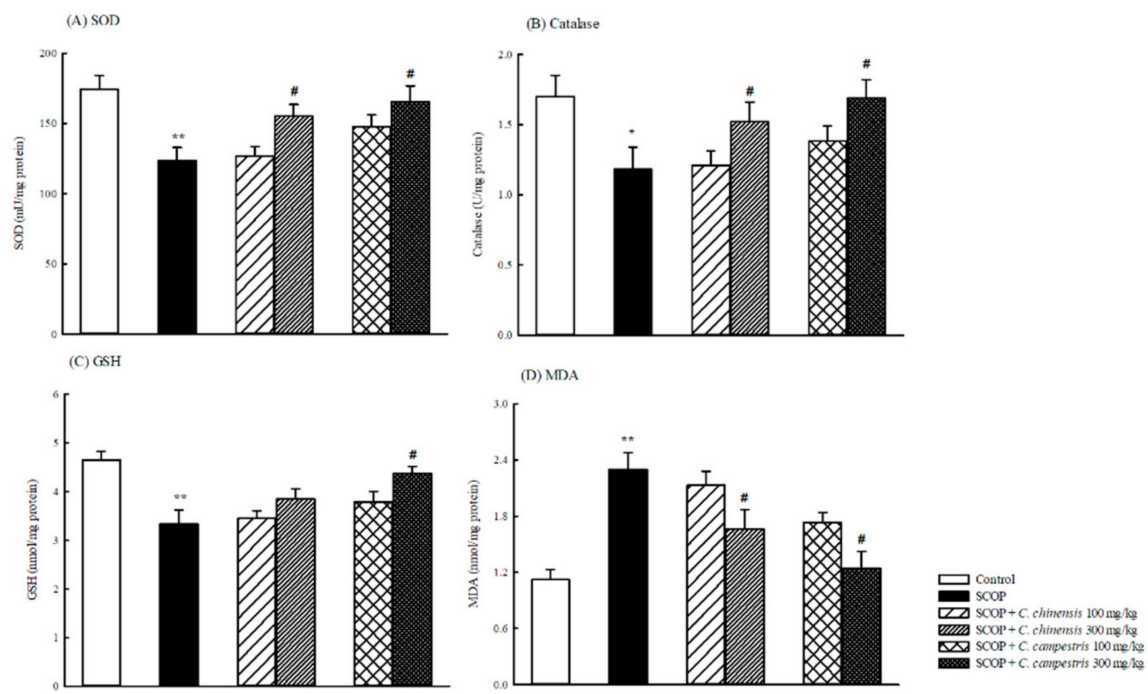

Figure 6. The effects of Cuscuta chinensis and C. campestris extracts (100 and $300 \mathrm{mg} / \mathrm{kg}$, po) on (A) brain superoxide dismutase (SOD) activities, (B) brain catalase activities, (C) brain glutathione (GSH) levels, and (D) brain malondialdehyde (MDA) levels in scopolamine (SCOP, $1 \mathrm{mg} / \mathrm{kg}$, ip)-treated mice. All the brain tissues were collected from the tested mice and then homogenized to obtain the total brain homogenates. The SOD and catalase activities and the GSH and MDA levels in the total brain homogenates were subsequently examined. Columns indicate mean $\pm \operatorname{SEM}(n=7) .{ }^{*} p<0.05$, ** $p<0.01$ compared with the control group. \# $p<0.05$ compared with the SCOP group. 


\subsection{Effects of C. chinensis and C. campestris Extracts on Brain Cytokine Levels in SCOP-Treated Mice}

SCOP increased brain interleukin-1 $\beta$ (IL-1 $\beta$ ) and tumor necrosis factor- $\alpha$ (TNF- $\alpha$ ) levels in mice (Figure 7). The C. chinensis extract at $300 \mathrm{mg} / \mathrm{kg}$ decreased brain IL-1 $\beta$ levels that were increased by SCOP injection (Figure 7). The C. campestris extract at $300 \mathrm{mg} / \mathrm{kg}$ decreased brain IL- $1 \beta$ and TNF- $\alpha$ levels that were increased by SCOP injection (Figure 7).

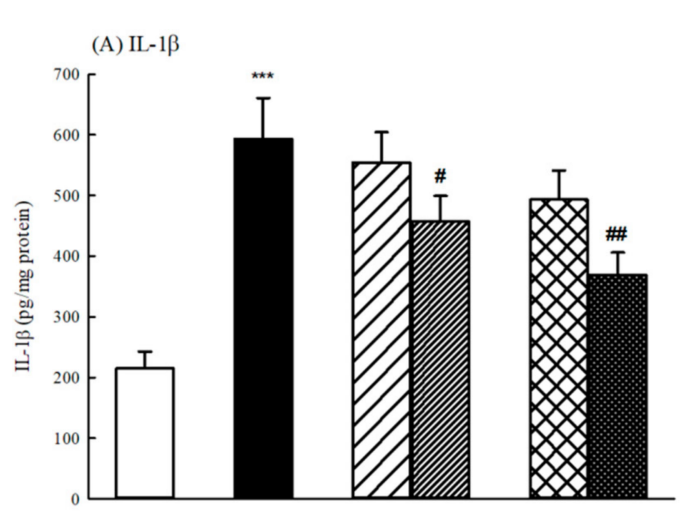

(B) TNF- $\alpha$
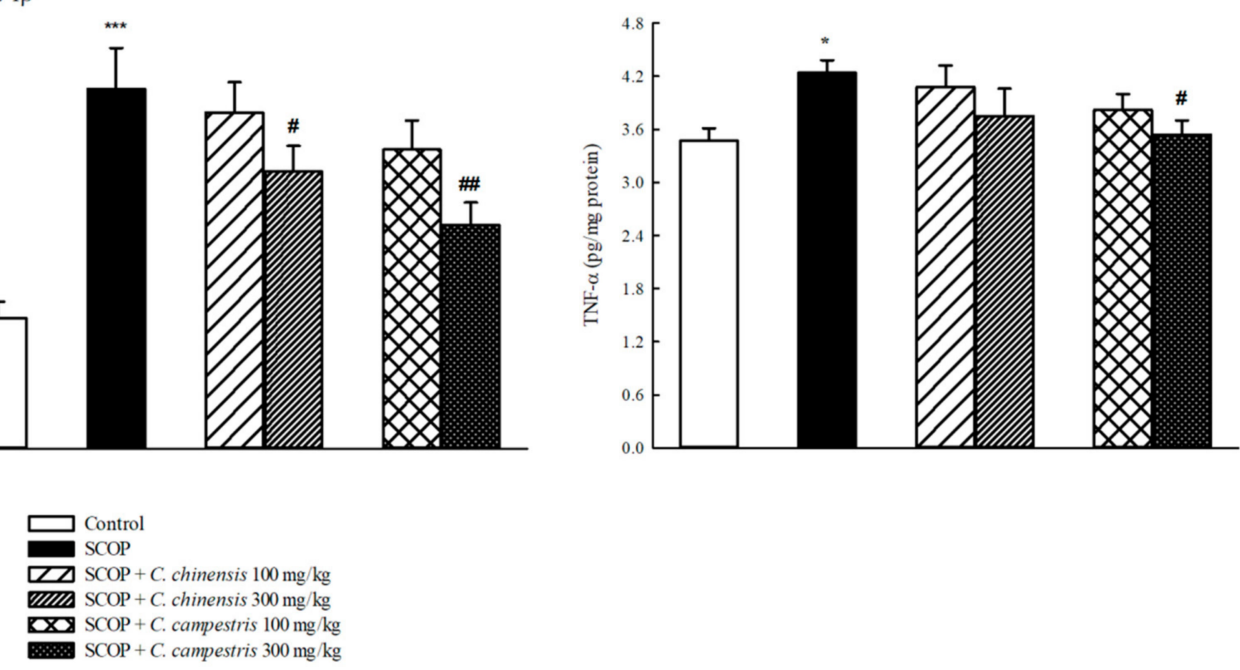

Figure 7. The effects of the Cuscuta chinensis and C. campestris extracts (100 and $300 \mathrm{mg} / \mathrm{kg}$, po) on (A) brain interleukin-1 $\beta$ (IL-1 $\beta$ ) levels and (B) brain tumor necrosis factor $\alpha$ (TNF- $\alpha$ ) levels in scopolamine (SCOP, $1 \mathrm{mg} / \mathrm{kg}$, ip)-treated mice. All the brain tissues were collected from the tested mice and then homogenized to obtain the total brain homogenates. The IL-1 $\beta$ and TNF- $\alpha$ levels in the total brain homogenates were subsequently examined. Columns indicate mean $\pm \operatorname{SEM}(n=7) .{ }^{*} p<0.05$, *** $p<0.001$ compared with the control group. \# $p<0.05$, \#\# $p<0.01$ compared with the SCOP group.

\subsection{AChE-Inhibiting Activities of C. chinensis and C. campestris In Vitro}

The AChE-inhibiting activities of $C$. chinensis and C. campestris were examined using the Ellman method. The results showed that $C$. chinensis and C. campestris extracts at $100-2000 \mu \mathrm{g} / \mathrm{mL}$ possess AChE-inhibiting activities, and C. campestris has a higher inhibitory activity (Figure 8).

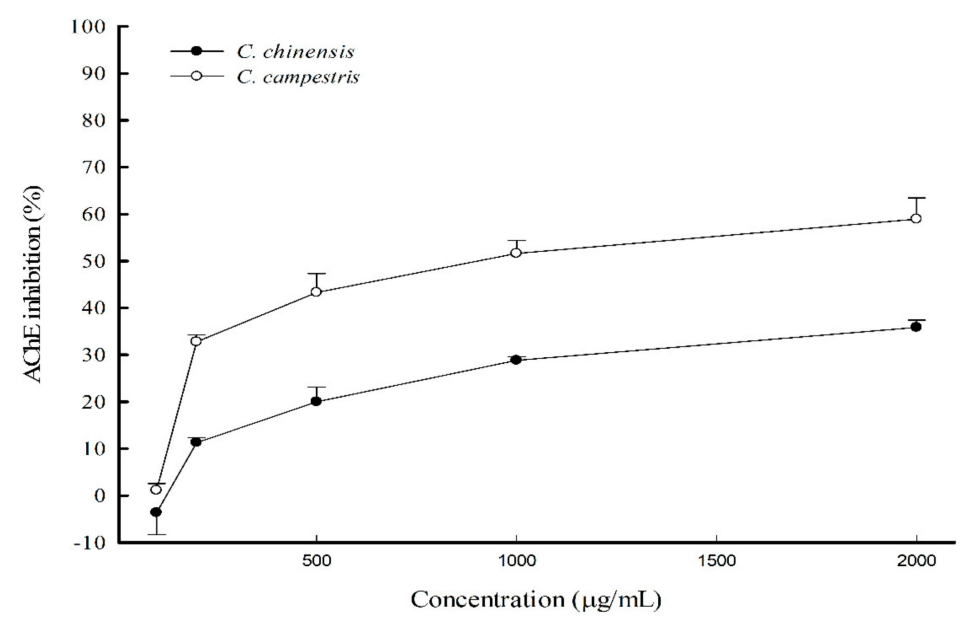

Figure 8. The acetylcholinesterase (AChE)-inhibiting effects of Cuscuta chinensis and C. campestris extracts in vitro. The supernatants of the brain homogenates were used as the AChE source for the assay. Four concentrations of the $C$. chinensis or $C$. campestris extract $(0.5,1,1.5$, and $2 \mathrm{mg} / \mathrm{mL})$ were used to examine their inhibitory activities. Data are expressed as mean $\pm \operatorname{SD}(n=3)$. 


\subsection{Antioxidant Compounds and Activity of C. chinensis and C. campestris In Vitro}

The total flavonoid content of $C$. chinensis and $C$. campestris extracts was determined to be $20.07 \pm 0.22$ and $28.34 \pm 0.95 \mathrm{mg} / \mathrm{g}$, respectively, which is equivalent to that of quercetin. Thus, C. campestris has higher flavonoid content than C. chinensis. The content of total phenolic compounds of C. chinensis and C. campestris extracts was $76.78 \pm 1.58$ and $65.11 \pm 0.21 \mathrm{mg} / \mathrm{g}$, respectively, which is equivalent to that of gallic acid. Thus, $C$. chinensis has a higher content of phenolic compounds than $C$. campestris (Table 1). Furthermore, the antioxidant capacities of C. chinensis and C. campestris extracts were examined through the 2,2'-azino-bis(3-ethylbenzothiazoline-6-sulphonic acid) (ABTS) decolorization assay and the diphenyl picrylhydrazyl (DPPH) scavenging assay. The results showed that they possess an antioxidant capacity, and C. chinensis exhibits a slightly higher activity than C. campestris (Table 1).

Table 1. The contents of total flavonoids and total phenolic compounds in the Cuscuta chinensis or C. campestris extract and their ABTS antioxidant capacity and DPPH scavenging ability.

\begin{tabular}{lcccc}
\hline & \multicolumn{2}{c}{ Contents of Antioxidant Compounds } & $\begin{array}{c}\text { Radical Scavenging Ability L-Ascorbic } \\
\text { Acid Equivalent }(\mu \mathrm{g} / \mathrm{mL})\end{array}$ \\
\hline & $\begin{array}{c}\text { Flavonoids }(\mathrm{mg} \\
\text { quercetin } \\
\text { equivalent/g) }\end{array}$ & $\begin{array}{c}\text { Total phenolics }(\mathrm{mg} \\
\text { gallic acid } \\
\text { equivalent/g) }\end{array}$ & ABTS & DPPH \\
\hline C. chinensis & $20.07 \pm 0.22$ & $76.78 \pm 1.58$ & $27.87 \pm 3.46$ & $11.18 \pm 0.46$ \\
C. campestris & $28.34 \pm 0.95$ & $65.11 \pm 0.21$ & $26.48 \pm 3.26$ & $10.80 \pm 0.48$ \\
\hline
\end{tabular}

Data are expressed as mean \pm SD $(n=3)$. ABTS: 2,2'-azino-bis(3-ethylbenzothiazoline-6-sulphonic acid), DPPH: diphenyl picrylhydrazyl.

\section{Discussion}

Traditional Chinese physicians commonly use C. chinensis as a herb for preventing aging. Early reports have indicated that $C$. chinensis improved memory impairment in the Y-maze or step-down avoidance test in aged, cerebral ischemia, or D-galactose-induced mice [22-24]. Due to the important role of the cerebral cholinergic system in memory function and AD, we newly discovered the memory-improving effects of $C$. chinensis and C. campestris on SCOP-induced memory deficit in mice and compared their efficacies simultaneously. In this study, this behavioral alteration caused by SCOP in mice was consistent with that described the previous reports in rodents $[10,25,26]$, indicating that SCOP-induced memory deficit in the passive avoidance test, elevated plus-maze, and MWM is related to hyperactivity (hyperlocomotion), poor attention (poor exploratory behavior), and subsequent learning acquisition impairment (poor memory function). The $C$. chinensis extract at $300 \mathrm{mg} / \mathrm{kg}$ improved the passive avoidance response, memory index in the elevated plus-maze, and spatial performance and reference memory in MWM in mice, which were impaired by SCOP. These results of the memory-improving effects of the extracts are consistent with the finding of other reports that $C$. chinensis restores memory function in various models such as models of D-galactose aging, natural aging, cerebral ischemia, and hypoendocrinism induced by removing bilateral ovaries [17,22-24]. The $C$. campestris extract at $300 \mathrm{mg} / \mathrm{kg}$ also improved these aforementioned memory deficits that were impaired by SCOP in mice. The memory-improving effect of the $C$. campestris extract on SCOP-induced memory deficits is slightly better than that of the C. chinensis extract in mice. However, neither the C. chinensis nor $C$. campestris extracts altered hyperactivities and poor attention that were caused by SCOP. Therefore, C. campestris exerts memory-improving effects on SCOP-induced memory deficits, and these two species mainly improve the memory deficit but not the behavioral alteration caused by SCOP.

According to the neuropathological characterization of $\mathrm{AD}$, cerebral oxidative stress and neuroinflammation are two major pathological factors that cause neuronal loss, particularly the degeneration of cholinergic neuronal circuits in BF [1]. Although SCOP is a muscarinic receptor antagonist, recent reports have indicated that SCOP causes oxidative damage and neuroinflammation in the cortex and hippocampus $[10,11]$. SCOP elevates oxidative modifications of proteins and lipids such as MDA and decreases the total antioxidant capacity in the brain [27]. SCOP also leads to the 
production of inflammatory factors, including IL-1 $\beta$ and TNF- $\alpha$, in the brain [28]. The present study demonstrated that SCOP decreased the activities of antioxidant enzymes, including SOD and catalase, and GSH levels in the brains of mice and then increased brain MDA levels. In addition, SCOP increased IL-1 $\beta$ and TNF- $\alpha$ levels in the brains of mice. The present data further indicate that SCOP elevates AChE activities in the brains of mice. Consistent with previous reports $[10,11,27]$, SCOP-induced memory deficits are strongly related to cholinergic dysfunction such as the upregulation of AChE activity and the blockade of muscarinic receptors, cerebral oxidative stress, and neuroinflammation. Some reports have stated that $C$. chinensis or $C$. campestris protects against oxidative damage caused by $\mathrm{H}_{2} \mathrm{O}_{2}$, acetaminophen, or $\mathrm{CCl}_{4}$ in vitro and in vivo [20,29,30]. C. chinensis or $C$. campestris possesses anti-inflammatory effects against LPS-induced or carrageenan-induced inflammation in vitro and in vivo $[13,15,16]$. The present study further explored the memory-improving mechanism of C. chinensis and C. campestris for SCOP-induced memory-impairment in mice. C. chinensis and C. campestris extracts only at $300 \mathrm{mg} / \mathrm{kg}$ restored the lower activities of the brain antioxidant defense system and decreased the higher brain MDA levels caused by SCOP in mice. C. chinensis and C. campestris extracts only at $300 \mathrm{mg} / \mathrm{kg}$ also decreased the higher brain IL-1 $\beta$ levels caused by SCOP in mice. Only the C. campestris extract at $300 \mathrm{mg} / \mathrm{kg}$ could restore brain GSH levels and decrease brain TNF- $\alpha$ levels in SCOP-injected mice. Only the C. campestris extract at $300 \mathrm{mg} / \mathrm{kg}$ inhibited the brain AChE activities upregulated by SCOP in mice. Furthermore, the present study also demonstrated that $C$. campestris possessed a higher AChE-inhibiting activity than $C$. chinensis in vitro. From these aforementioned results, we confirm that $C$. chinensis and $C$. campestris can improve the memory impairment caused by SCOP by restoring cholinergic function and decreasing oxidative stress and neuroinflammation. C. campestris has a higher memory-improving potential than $C$. chinensis against SCOP-induced memory impairment.

Previous studies have shown that seeds of both $C$. chinensis and C. campestris possess anti-inflammatory and antiproliferative activities, and the seeds of $C$. campestris exhibit a higher activity [16]. In this study, we demonstrated that C. campestris has a higher flavonoid content than C. chinensis, whereas C. chinensis has a higher content of phenolic compounds than C. campestris. The seeds of $C$. chinensis exhibit a higher antioxidant capacity than those of $C$. campestris. Therefore, the phenolic compounds in Cuscuta seeds make great contributions to the antioxidant capacity. However, C. campestris exhibits higher anti-inflammatory, antiproliferative [16], and neuroprotective bioactivities. Moreover, C. campestris has a higher content of flavonoids than C. chinensis. Therefore, these findings indicate that the flavonoids in the phenolic compounds make great contributions for the above bioactivities. Interestingly, quercetin accumulates at a dramatically higher level (about $2.14 \mathrm{mg} / \mathrm{g}$ ) in the seeds of C. campestris than in C. chinensis [16]. In addition, quercetin possesses higher AChE-inhibiting potency in vitro and augments manganese-induced neurobehavioral alteration through the restoration of brain antioxidant status and AChE activity in rats [31-33]. These results indicate that quercetin may play a critical role in neuroprotective bioactivity in vitro and in vivo.

\section{Materials and Methods}

\subsection{Preparation of Plant Extract}

C. chinensis seeds were purchased from a Chinese medicine hospital (Union Traditional Medical Hospital, Taichung, Taiwan). C. campestris seeds were collected from C. campestris plants grown on Bidens pilosa L. var. radiata Sch. Bip. in the wild (Taichung, Taiwan). The seeds of both species were authenticated by Wen-Huang Peng and Ming-Kuem Lin (both from the Department of Chinese Pharmaceutical Sciences and Chinese Medicine Resources, China Medical University, Taichung, Taiwan), who observed the morphology of the flowers and confirmed the chemical compositions of the seeds $[13,16,20]$. The raw seeds were processed as described previously [16]. To prepare methanol extracts, Cuscuta seeds were extracted with methanol through sonication, as described previously [16]. Phytochemical profiles of $C$. chinensis and C. campestris methanol extracts were determined and compared to confirm their identities (Figure 9). 


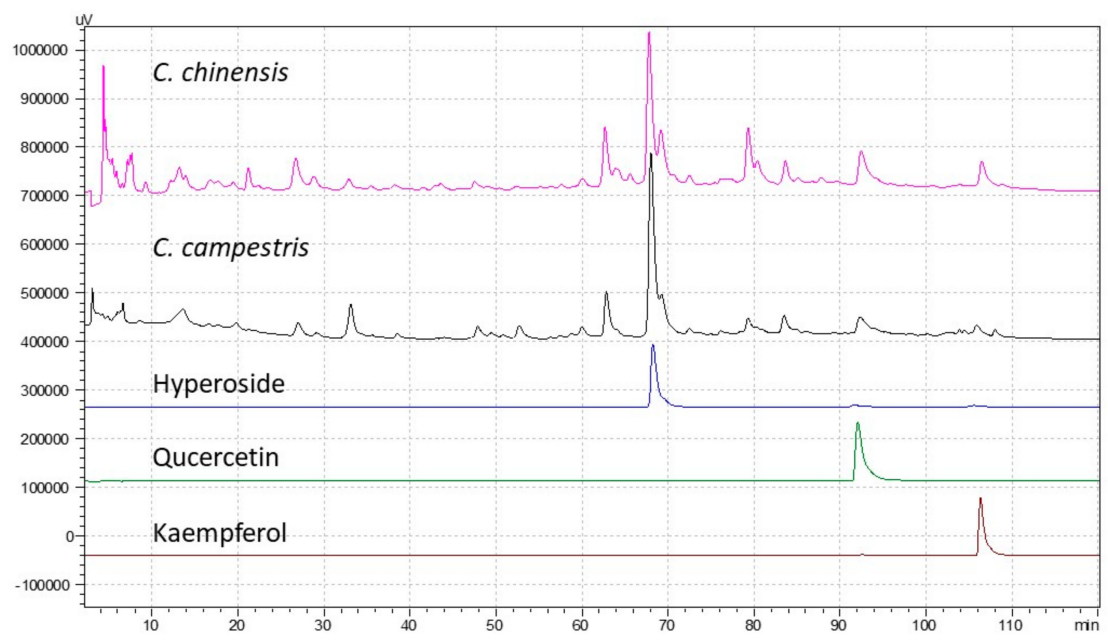

Figure 9. The HPLC profiles of C. chinensis and C. campestris. Phytochemical analyses of C. chinensis and $C$. campestris methanol extracts, as well as three standards, were performed using a Shimadzu HPLC system with an RP-18 column at UV $254 \mathrm{~nm}$.

\subsection{Chemicals}

The reagents 5,5'-dithio-bis(2-nitrobenzoic) acid (DTNB), ABTS, AChE, acetylthiocholine iodide (ACtCh), aluminum chloride, L-ascorbic acid, DPPH, Folin-Ciocalteu's reagent, gallic acid, GSH, glutathione reductase, nitroblue tetrazolium chloride (NBT), hyperoside, quercetin, kaempferol, SCOP hydrobromide, SOD, sodium carbonate, trichloroacetic acid, thiobarbituric acid (TBA), tetraisopropyl pyrophosphoramide (iso-OMPA), xanthine oxidase, and xanthine were purchased from Sigma-Aldrich Chem. Corp. (St. Louis, MO, USA).

\subsection{HPLC Analysis}

Samples and standards were resolved in methanol and filtered through a 0.22-mm membrane. HPLC was performed on a Shimadzu HPLC system (Kyoto, Japan) equipped with a Shimadzu LC-20AT pump, a Shimadzu SIL-20 autosampler, and a Shimadzu SPD-M20A detector (wavelength: 190-800 nm). The HPLC profiles of C. chinensis and C. campestris were determined using an RP-18 column (Cosmosil C18, $4.6 \times 250 \mathrm{~mm}, 5 \mathrm{~mm}$, Nacalai Tesque Inc., Kyoto, Japan) at a flow rate of $1.0 \mathrm{~mL} / \mathrm{min}$, detected at UV $254 \mathrm{~nm}$. The injection volume was $10 \mu \mathrm{L}$. The mobile phase was composed of $0.1 \%$ TFA water solution (solvent A) and methanol (solvent B). The solvent gradient was as follows: $0-120 \mathrm{~min}$ from $10 \%$ to $60 \%$ solvent $\mathrm{B}$.

\subsection{Animals}

Eight-week-old male ICR mice (20-25 g) were obtained from BioLASCO Taiwan Co., Ltd. (Yi-Lan, Taiwan). They were housed randomly in groups of six at a temperature of $23^{\circ} \mathrm{C} \pm 1{ }^{\circ} \mathrm{C}, 60 \%$ humidity, and 12/12-h light/dark cycle (light phase: 08:00 to 20:00). These mice were maintained following the Guiding Principles for the Care and Use of Laboratory Animals, and the experimental protocol was approved by the Institutional Animal Care and Use Committee of China Medical University (CMUIACUC-2017392). After acclimatization for 1 week, normal mice were used in the experiments mentioned below.

\subsection{Drug Treatment and Group Division}

The schedule of drug treatments and behavioral measurements is shown in Figure 10. SCOP $(1 \mathrm{mg} / \mathrm{kg}$ ) was intraperitoneally (ip) injected into mice $30 \mathrm{~min}$ before behavioral measurements [26]. The C. chinensis or C. campestris extract $(100$ and $300 \mathrm{mg} / \mathrm{kg}$ ) was orally administered to mice $1 \mathrm{~h}$ before behavioral measurements. Behavioral measurements were performed in the following order: locomotor 
and exploratory tests, passive avoidance test, elevated plus-maze, and spatial performance test and probe test of MWM. On the next day, the probe test was conducted, and the tested mice were sacrificed $1 \mathrm{~h}$ after the test. The activities of AChE and antioxidant enzymes (SOD and catalase) and the levels of GSH, MDA, and cytokines (IL-1 $\beta$ and TNF- $\alpha$ ) were monitored in the brain tissues of the tested mice.

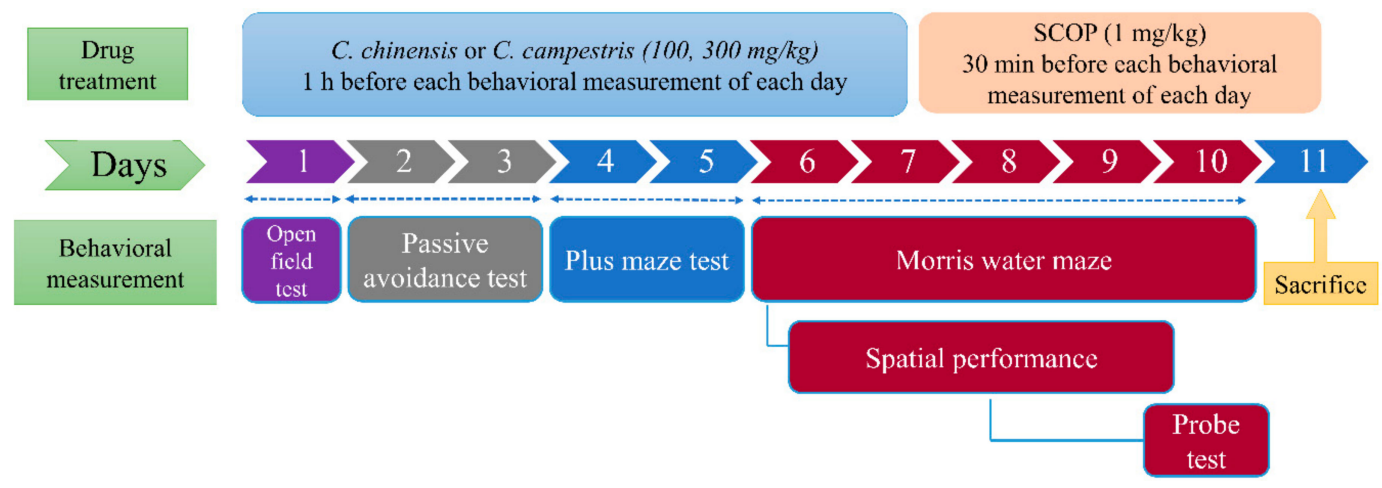

Figure 10. The schedule of drug treatments and behavioral measurements. The Cuscuta chinensis or C. campestris extract (100 and $300 \mathrm{mg} / \mathrm{kg}$, po) was administered $1 \mathrm{~h}$ before each behavioral assessment of each day during the experimental period. Scopolamine (SCOP, $1 \mathrm{mg} / \mathrm{kg}$, ip) was administered $1 \mathrm{~h}$ before each behavioral assessment of each day during the experimental period. C. chinensis: Cuscuta chinensis; C. campestris: Cuscuta campestris.

\subsection{Locomotor and Exploratory Tests}

The open field apparatus (Coulbourn Instruments L.L.C., Holliston, MA, USA) was used to perform the locomotor and exploratory tests simultaneously. Each mouse was placed in the open field apparatus; then, the movement time, distance, velocity, number of entries, and time spent in the hole were recorded for $10 \mathrm{~min}$ by using the TruScan software v 2.07 (Coulbourn Instruments L.L.C., Holliston, MA, USA) [25].

\subsection{Passive Avoidance Response}

Two compartments (light and dark compartments) of the same size that had a steel-rod grid floor (Coulbourn Instruments L.L.C., Holliston, MA, USA) were used to perform the passive avoidance test. A guillotine door was located between the two compartments. In the beginning, each mouse was placed in the light compartment; then, the STL was measured by recording the time when the door was opened and the mouse entered the dark compartment. The door was locked once the mouse entered the dark compartment. Subsequently, the mouse received a footshock through the steel rods. The mouse was placed back its home cage $5 \mathrm{~s}$ after the shock. After $24 \mathrm{~h}$, the same experiment was conducted again, and the STL for the second day was recorded [26].

\subsection{Elevated Plus-Maze}

Two enclosed arms and two open arms were connected with each other to form a plus-maze. The plus-maze was elevated to a height of $0.5 \mathrm{~m}$ above the floor. In the acquisition session, each mouse was placed at the distal end of an open arm, and the TL was recorded. After entering the enclosed arm, the mouse was free to move in the maze for $10 \mathrm{~s}$. Subsequently, the mouse returned to its home cage. The retention session was performed $24 \mathrm{~h}$ after the acquisition session [34]. The TL in the acquisition and retention sessions were recorded by a video camera equipped with an automated video tracking system device and EthoVision XT software (version 10, Noldus Information Technology, Leesburg, VA, USA).

\section{9. $M W M$}

A black circular stainless pool containing water $\left(23^{\circ} \mathrm{C} \pm 1{ }^{\circ} \mathrm{C}\right)$ to a depth of $35 \mathrm{~cm}$ and a hidden platform submerged $1.0 \mathrm{~cm}$ below the water was used for MWM. In four sessions for 4 days, 
each mouse was trained each day to find the hidden platform in the spatial performance test. During the training, the mouse facing the pool wall was placed in the water and was allowed to find the platform within $60 \mathrm{~s}$. If the mouse reached the platform successfully, it was allowed to rest for $15 \mathrm{~s}$ on the platform. If it could not reach the platform within $60 \mathrm{~s}$, the mouse was given a score of $60 \mathrm{~s}$, was moved manually to the platform and was allowed to rest for $15 \mathrm{~s}$ on the platform. Each mouse was trained four times. The swimming path and escape latency to the platform were also recorded using the aforementioned video camera [25]. On the next day, the platform was removed, and the reference memory was then measured by performing the probe test. In addition, each mouse was placed into the pool at the location opposite to the previous location. The distance moved and time spent by each mouse in each quadrant was recorded during the $60 \mathrm{~s}$ [25].

\subsection{Brain Tissue Preparation}

All mice were anesthetized with sodium pentobarbital $(50 \mathrm{mg} / \mathrm{kg})$ and were sacrificed through decapitation in an unconscious state after behavioral measurements. All brain tissues were collected and homogenized with $9 \times(w / v)$ ice-cold phosphate buffered saline. The homogenates were centrifuged at $10,000 \times \mathrm{g}$ for $15 \mathrm{~min}$ at $4{ }^{\circ} \mathrm{C}$, and the aliquots of supernatants were stored at $-80^{\circ} \mathrm{C}$ until the examination of $\mathrm{AChE}$ and antioxidant enzyme activities and GSH and MDA levels.

\subsection{Measurement of Brain AChE Activities}

Brain AChE activity was determined through a method described in a previous report [25]. The supernatant solution or AChE standard solution was incubated with DTNB at $25^{\circ} \mathrm{C}$ for $10 \mathrm{~min}$; then, $0.3 \mathrm{mM}$ ACtCh was added for color development. The yellow product was measured at $412 \mathrm{~nm}$ using a spectrophotometric microplate reader (PowerWave X340, Bio-Tek instruments, Inc., Winooski, VT, USA). Brain AChE activity is expressed as $\mathrm{U} A C h E / m g$ of protein.

\subsection{Measurement of Brain Antioxidant Enzyme Activities}

Catalase and SOD activities and GSH and MDA levels were determined using a spectrophotometric microplate reader [35]. Catalase activity was examined by measuring the absorbance of Amplex Red at $560 \mathrm{~nm}$. SOD activity was determined by measuring the absorbance of NBT at $560 \mathrm{~nm}$. Catalase and SOD activities were expressed as U/mg of protein. The GSH level was determined by measuring the absorbance of DTNB at $405 \mathrm{~nm}$. The GSH levels are expressed as $\mathrm{nmol} / \mathrm{mg}$ of protein. Brain MDA levels were determined through the TBARS assay. The absorbance of TBA was determined at $532 \mathrm{~nm}$. MDA levels are expressed as nmol/mg of protein.

\subsection{Measurement of Brain IL-1 $\beta$ and TNF- $\alpha$ Levels}

The brain tissues were homogenized with a lysis solution containing protease inhibitors $(0.4 \mathrm{M}$ $\mathrm{NaCl}, 0.1 \mathrm{mM}$ phenylmethylsulfonyl fluoride, $0.1 \mathrm{mM}$ benzethonium chloride, $10 \mathrm{mM}$ EDTA, $0.5 \%$ bovine serum albumin, $0.05 \%$ Tween 20 , and $10 \mu \mathrm{g} / \mathrm{mL}$ aprotinin). Homogenates were centrifuged at $10,000 \times g$ at $4{ }^{\circ} \mathrm{C}$, and the supernatants were stored at $-80^{\circ} \mathrm{C}$ until the following analyses. The levels of TNF- $\alpha$ and IL- $1 \beta$ proteins were determined using ELISA kits (R\&D Systems, Abingdon, UK) according to the manufacturer's protocol.

\subsection{In Vitro AChE-Inhibiting Activity Assay}

The assay of AChE-inhibiting activity was conducted as described in a previous report [26]. The mouse brain was dissected on ice and homogenized. The brain homogenate was centrifuged, and the supernatant was used as the AChE source for the assay. Four concentrations of the $C$. chinensis or C. campestris extract $(0.5,1,1.5$, and $2 \mathrm{mg} / \mathrm{mL})$ were used to examine their inhibitory activities. The reaction mixture was placed in a Bio-Teck PowerWave 340X microplate reader (Bio-Tek instruments, 
Inc., Winooski, VT, USA) to record the absorbance at $412 \mathrm{~nm}$. The percentage of AChE inhibition was calculated by comparing the rates of the samples to those of the solvent.

\subsection{Measurement of Antioxidant Phytoconstituent Contents and Activity In Vitro}

The total flavonoid content in the extract was determined using the aluminum chloride colorimetric assay [35]. The content of the total phenolic compounds in the extract was estimated using the Folin-Ciocalteu method [35]. The ABTS decolorization assay and DPPH scavenging assay were used to measure the antioxidant capacity in comparison with the standard L-ascorbic acid [35].

\subsection{Statistical Analyses}

All values are expressed as the mean \pm standard error of the mean (SEM). IBM SPSS version 20.0 (IBM, Chicago, IL, USA) was used to analyze all the results through one-way ANOVA followed by Scheffe's test. A $p$ value of $<0.05$ was considered significant.

\section{Conclusions}

In Chinese medicine pharmacopoeias, Cuscutae Semen mainly refers to the seeds of $C$. chinensis Lam. According to the findings of previous reports for anti-inflammatory and antiproliferative activities [16] and the present results for antioxidant and neuroprotective capacities, the seeds of C. campestris exhibit the same bioactivities as those of $C$. chinensis. The memory-improving mechanism against SCOP-induced memory impairment is mainly entered by restoring the cholinergic function and decreasing oxidative stress and neuroinflammation. Therefore, the seeds of $C$. campestris can be used as Cuscutae Semen in Traditional Chinese Medicine.

Author Contributions: Conceptualization, C.-R.W. and M.-K.L.; methodology, C.-R.W.; investigation, H.-C.H., T.-J.C. and Y.-D.C.; resources, M.-S.L. and M.-K.L.; writing-original draft preparation, M.-S.L. and M.-K.L.; writing-review and editing, C.-R.W. supervision, C.-R.W.

Funding: This research was funded by the Ministry of Science and Technology, grant number MOST 105-2320-B-039-012-MY3.

Conflicts of Interest: The authors declare no conflict of interest. The funders had no role in the design of the study; in the collection, analyses, or interpretation of data; in the writing of the manuscript, or in the decision to publish the results.

\section{Abbreviations}

$\begin{array}{ll}\text { ABTS } & \text { 2,2'-azino-bis(3-ethylbenzothiazoline-6-sulphonic acid) } \\ \text { AChE } & \text { acetylcholinesterase } \\ \text { ACtCh } & \text { acetylthiocholine iodide } \\ \text { AD } & \text { Alzheimer's disease } \\ \text { BF } & \text { basal forebrain } \\ \text { DMSO } & \text { dimethyl sulfoxide } \\ \text { DPPH } & \text { diphenyl picrylhydrazyl } \\ \text { DTNB } & \text { 5,5'-dithio-bis(2-nitrobenzoic) acid } \\ \text { GPx } & \text { glutathione peroxidase } \\ \text { GR } & \text { glutathione reductase } \\ \text { GSH } & \text { glutathione } \\ \text { iso-OMPA } & \text { tetraisopropyl pyrophosphoramide } \\ \text { MDA } & \text { malondialdehyde } \\ \text { MWM } & \text { Morris water maze } \\ \text { NBT } & \text { nitroblue tetrazolium chloride } \\ \text { SCOP } & \text { scopolamine } \\ \text { SOD } & \text { superoxide dismutase } \\ \text { TBA } & \text { thiobarbituric acid } \\ \text { TCA } & \text { trichloroacetic acid }\end{array}$




\section{References}

1. Karran, E.; De Strooper, B. The amyloid cascade hypothesis: Are we poised for success or failure? J. Neurochem. 2016, 139 (Suppl. 2), 237-252. [CrossRef]

2. Pohanka, M. Alzheimer s disease and oxidative stress: A review. Curr. Med. Chem. 2014, 21, 356-364. [CrossRef] [PubMed]

3. Omar, R.A.; Chyan, Y.J.; Andorn, A.C.; Poeggeler, B.; Robakis, N.K.; Pappolla, M.A. Increased expression but reduced activity of antioxidant enzymes in Alzheimer's disease. J. Alzheimers Dis. 1999, 1, 139-145. [CrossRef] [PubMed]

4. Serrano-Pozo, A.; Frosch, M.P.; Masliah, E.; Hyman, B.T. Neuropathological alterations in Alzheimer disease. Cold Spring Harb. Perspect. Med. 2011, 1. [CrossRef] [PubMed]

5. Querfurth, H.W.; LaFerla, F.M. Alzheimer's disease. N Engl. J. Med. 2010, 362, 329-344. [CrossRef] [PubMed]

6. Jodar, L.; Kaneto, H. Synaptic plasticity: Stairway to memory. Jpn. J. Pharmacol. 1995, 68, 359-387. [CrossRef] [PubMed]

7. Myhrer, T. Neurotransmitter systems involved in learning and memory in the rat: A meta-analysis based on studies of four behavioral tasks. Brain Res. Rev. 2003, 41, 268-287. [CrossRef]

8. Okaichi, H.; Oshima, Y.; Jarrard, L.E. Scopolamine impairs both working and reference memory in rats: A replication and extension. Pharmacol. Biochem. Behav. 1989, 34, 599-602. [CrossRef]

9. Elrod, K.; Buccafusco, J.J. An evaluation of the mechanism of scopolamine-induced impairment in two passive avoidance protocols. Pharmacol. Biochem. Behav. 1988, 29, 15-21. [CrossRef]

10. Kim, M.S.; Lee, D.Y.; Lee, J.; Kim, H.W.; Sung, S.H.; Han, J.S.; Jeon, W.K. Terminalia chebula extract prevents scopolamine-induced amnesia via cholinergic modulation and anti-oxidative effects in mice. BMC Complement. Altern. Med. 2018, 18, 136. [CrossRef] [PubMed]

11. Demirci, K.; Naziroglu, M.; Ovey, I.S.; Balaban, H. Selenium attenuates apoptosis, inflammation and oxidative stress in the blood and brain of aged rats with scopolamine-induced dementia. Metab. Brain Dis. 2017, 32, 321-329. [CrossRef] [PubMed]

12. Ahmad, A.; Tandon, S.; Xuan, T.D.; Nooreen, Z. A Review on phytoconstituents and biological activities of Cuscuta species. Biomed. Pharm. 2017, 92, 772-795. [CrossRef] [PubMed]

13. Liao, J.C.; Chang, W.T.; Lee, M.S.; Chiu, Y.J.; Chao, W.K.; Lin, Y.C.; Lin, M.K.; Peng, W.H. Antinociceptive and anti-inflammatory activities of Cuscuta chinensis seeds in mice. Am. J. Chin. Med. 2014, 42, 223-242. [CrossRef] [PubMed]

14. Donnapee, S.; Li, J.; Yang, X.; Ge, A.H.; Donkor, P.O.; Gao, X.M.; Chang, Y.X. Cuscuta chinensis Lam.: A systematic review on ethnopharmacology, phytochemistry and pharmacology of an important traditional herbal medicine. J. Ethnopharmacol. 2014, 157, 292-308. [CrossRef] [PubMed]

15. Kang, S.Y.; Jung, H.W.; Lee, M.Y.; Lee, H.W.; Chae, S.W.; Park, Y.K. Effect of the semen extract of Cuscuta chinensis on inflammatory responses in LPS-stimulated BV-2 microglia. Chin. J. Nat. Med. 2014, 12, 573-581. [CrossRef]

16. Lee, M.S.; Chen, C.J.; Wan, L.; Koizumi, A.; Chang, W.T.; Yang, M.J.; Lin, W.H.; Tsai, F.J.; Lin, M.K. Quercetin is increased in heat-processed Cuscuta campestris seeds, which enhances the seed's anti-inflammatory and anti-cancer activities. Process. Biochem. 2001, 46, 2248-2254. [CrossRef]

17. Peng, S.M.; Chen, Q.; Chen, Y.; Ye, H.; Wang, T. The protective mechanism of total flavonoids from Semen Cuscutae (TFSC) on learning-memory function in Alzheimer's disease mice with hypoendocrinism. Acta Laser Biol. Sinica 2014, 23, 218-226.

18. Yang, D.; Wang, G.M.; Zhai, H.Y. Effects of Cuscuta chinensis flavonoids on inflammatory response of brain tissue in rats with cerebral ischemia-reperfusion injury. Zhongguo Yaofang 2013, 24, 979-982.

19. Jakovljević, D.V.; Vrvić, M.M.; Vrbničanin, S.; Sarić-Krsmanović, M. Phytochemical, free radical scavenging and antifungal profile of Cuscuta campestris Yunck. Chem. biodiversity 2018, 15. [CrossRef] [PubMed]

20. Peng, W.H.; Chen, Y.W.; Lee, M.S.; Chang, W.T.; Tsai, J.C.; Lin, Y.C.; Lin, M.K. Hepatoprotective effect of Cuscuta campestris Yunck. whole plant on carbon tetrachloride induced chronic liver injury in mice. Int. J. Mol. Sci. 2016, 17. [CrossRef] [PubMed]

21. Ye, M.; Yan, Y.N.; Qiao, L.; Ni, X.M. Studies on chemical constituents of Cuscuta chinensis. China J. Chin. Mater. Med. 2002, 27, 115-117. 
22. Ho, G.G.; Shi, W.; Liu, H.Y. The anti-aging effects of the extracts of Cuscuta chinensis in D-galactose-induced aging mice. Chin. J. Gerontol. 2015, 35, 5444-5446.

23. Lan, H.; Du, S.M. Study on the anti-aging action of the extrects of Cuscuta chinensis in natural aging mice. Chin. Pharm. 2010, 21, 3667-3669.

24. Ji, Z.H.; Zhang, X.L.; Dong, L.F. Effect of the water extract of Cuscuta chinensis Lam. on memory impairment in cerebral ischemic rats. Chin. J. Behav. Med. Sci. 2006, 15, 681-682.

25. Shiao, Y.J.; Su, M.H.; Lin, H.C.; Wu, C.R. Acteoside and isoacteoside protect amyloid beta peptide induced cytotoxicity, cognitive deficit and neurochemical disturbances in vitro and in vivo. Int. J. Mol. Sci. 2017, 18. [CrossRef] [PubMed]

26. Wu, C.R.; Chang, C.L.; Hsieh, P.Y.; Lin, L.W.; Ching, H. Psoralen and isopsoralen, two coumarins of Psoraleae Fructus, can alleviate scopolamine-induced amnesia in rats. Planta Med. 2007, 73, 275-278. [CrossRef] [PubMed]

27. Skalicka-Wozniak, K.; Budzynska, B.; Biala, G.; Boguszewska-Czubara, A. Scopolamine-Induced memory impairment is alleviated by xanthotoxin: Role of acetylcholinesterase and oxidative stress processes. ACS Chem. Neurosci. 2018, 9, 1184-1194. [CrossRef] [PubMed]

28. Ahmad, A.; Ramasamy, K.; Jaafar, S.M.; Majeed, A.B.; Mani, V. Total isoflavones from soybean and tempeh reversed scopolamine-induced amnesia, improved cholinergic activities and reduced neuroinflammation in brain. Food Chem. Toxicol. 2014, 65, 120-128. [CrossRef] [PubMed]

29. Yen, F.L.; Wu, T.H.; Lin, L.T.; Lin, C.C. Hepatoprotective and antioxidant effects of Cuscuta chinensis against acetaminophen-induced hepatotoxicity in rats. J. Ethnopharmacol. 2007, 111, 123-128. [CrossRef] [PubMed]

30. Zhen, G.H.; Jiang, B.; Bao, Y.M.; Li, D.X.; An, L.J. The protect effect of flavonoids from Cuscuta chinensis in PC12 cells from damage induced by $\mathrm{H}_{2} \mathrm{O}_{2}$. J. Chin. Med. Mater. 2006, 29, 1051-1055.

31. Olennikov, D.N.; Kashchenko, N.I.; Chirikova, N.K.; Akobirshoeva, A.; Zilfikarov, I.N.; Vennos, C. Isorhamnetin and Quercetin Derivatives as Anti-Acetylcholinesterase Principles of Marigold (Calendula officinalis) Flowers and Preparations. Int. J. Mol. Sci. 2017, 18. [CrossRef] [PubMed]

32. Adedara, I.A.; Ego, V.C.; Subair, T.I.; Oyediran, O.; Farombi, E.O. Quercetin Improves Neurobehavioral Performance Through Restoration of Brain Antioxidant Status and Acetylcholinesterase Activity in Manganese-Treated Rats. Neurochem. Res. 2017, 42, 1219-1229. [CrossRef] [PubMed]

33. Ademosun, A.O.; Oboh, G.; Bello, F.; Ayeni, P.O. Antioxidative Properties and Effect of Quercetin and Its Glycosylated Form (Rutin) on Acetylcholinesterase and Butyrylcholinesterase Activities. J. Evid. Based Complement. Altern. Med. 2016, 21. [CrossRef] [PubMed]

34. Da Cunha, I.C.; Jose, R.F.; Orlandi Pereira, L.; Pimenta, J.A.; Oliveira de Souza, I.A.; Reiser, R.; Moreno, H., Jr.; Marino Neto, J.; Paschoalini, M.A.; Faria, M.S. The role of nitric oxide in the emotional learning of rats in the plus-maze. Physiol. Behav. 2005, 84, 351-358. [CrossRef] [PubMed]

35. Wu, C.R.; Chang, H.C.; Cheng, Y.D.; Lan, W.C.; Yang, S.E.; Ching, H. Aqueous Extract of Davallia mariesii Attenuates 6-Hydroxydopamine-Induced Oxidative Damage and Apoptosis in B35 Cells Through Inhibition of Caspase Cascade and Activation of PI3K/AKT/GSK-3 $\beta$ Pathway. Nutrients 2018, 10. [CrossRef] [PubMed]

Sample Availability: Not available. 